\title{
On the Measure of Totally Real Algebraic Integers. II
}

\author{
By C. J. Smyth
}

\begin{abstract}
For a certain measure $\Omega(\alpha)$, defined for totally real algebraic integers $\alpha \neq 0, \pm 1$, we find the four smallest values of $\Omega(\alpha)$. The methods used involve linear programming, and the results are verified using Interval Arithmetic.
\end{abstract}

1. Introduction. Let $\alpha \neq 0, \pm 1$ be a totally real algebraic integer of degree $d$, with conjugates $\alpha=\alpha_{1}, \ldots, \alpha_{d}$, and put $\Omega(\alpha)=\left(\prod_{i=1}^{d} \max \left(1,\left|\alpha_{i}\right|\right)\right)^{1 / d}$, a quantity which measures the 'average' size of the conjugates of $\alpha$. Denoting by $\mathcal{L}$ the set of all such $\Omega(\alpha)$, we showed in [1] that, from a result of Schinzel, $\mathcal{L}$ has least element $\left(\frac{1}{2}(1+\sqrt{ } 5)\right)^{1 / 2} \approx 1.2720$, and furthermore that there is a number $\ell \approx 1.31427$ such that $\mathcal{L}$ is dense in $(l, \infty)$.

Towards determining the structure of $\mathcal{E}$ in the gap $\left(\left(\frac{1}{2}(1+\sqrt{ } 5)\right)^{1 / 2}, \ell\right)$, we prove the following

THEOREM. The four smallest elements of $\mathcal{L}$ are $\Omega\left(\beta_{1}\right) \approx 1.2720, \Omega\left(\beta_{2}\right) \approx 1.2982$, $\Omega\left(\beta_{3}\right) \approx 1.3077$, and $\Omega\left(\alpha_{7}\right) \approx 1.3098$, where $\beta_{1}, \beta_{2}, \beta_{3}$ and $\alpha_{7}=2 \cos (2 \pi / 7)$ have minimal polynomials $x^{2}-x-1, x^{4}-x^{3}-3 x^{2}+x+1, x^{8}-x^{7}-7 x^{6}+4 x^{5}+$ $13 x^{4}-4 x^{3}-7 x^{2}+x+1$, and $x^{3}+x^{2}-2 x-1$, respectively.

The $\beta_{i}$ were defined in [1] using the operator $H$, given by $H x=x-x^{-1}$, as follows: $\beta_{0}=1$ and $\beta_{i}>1$ satisfies $H \beta_{i}=\beta_{i-1}(i>1)$. Furthermore, $H\left(H\left(H\left(\alpha_{7}\right)\right)\right)=-\alpha_{7}$, and there are other $\alpha$ with $\Omega(\alpha)$ small which are associated with fixed points of iterates of $H$; see [1, Section 6].

2. Setting Up the Problem. The principle of the proof of the theorem is a simple one: we make a list of $n$ totally positive algebraic integers $\alpha^{\prime}$ we know of which have small values of $\Omega\left(\alpha^{\prime}\right)$. Suppose these $\alpha^{\prime}$ have minimal polynomials $P_{1}, \ldots, P_{n}$. Then for any totally positive $\alpha$ not on the list, the resultant of $\alpha$ and $\alpha^{\prime}$ is a nonzero integer, so that

$$
\prod_{i=1}^{d}\left|P_{j}\left(\alpha_{i}\right)\right| \geqslant 1 \quad(j=1, \ldots, n)
$$

where the $\alpha_{i}$ are the conjugates of $\alpha$. Writing

$$
\mu_{\alpha}(x)=\frac{1}{d} \times \text { number of } \alpha_{i} \text { in }(0, x]
$$

Received May 27, 1980; revised January 15, 1981.

1980 Mathematics Subject Classification. Primary 12A95, 12B05.

(C) 1981 American Mathematical Society 0025-5718/81/0000-0117/\$02.00 
we can express (1) as

$$
\int_{0}^{\infty} \log \left|P_{j}(x)\right| d \mu_{\alpha}(x) \geqslant 0 \quad(i=1, \ldots, n) .
$$

Furthermore,

$$
\log \Omega(\alpha)=\int_{0}^{\infty} \log _{+} x d \mu_{\alpha}(x)
$$

where $\log _{+} x=\max (0, \log x)$.

We therefore pose the following programming problem for a general distribution $\mu(x)$ on the nonnegative real line $(\mu(0)=0, \mu(\infty)=1, \mu$ nondecreasing):

$$
\left\{\begin{array}{l}
\text { Minimize } y=\int_{0}^{\infty} \log _{+} x d \mu(x) \\
\text { subject to } \int_{0}^{\infty} \log \left|P_{j}(x)\right| d \mu(x) \geqslant 0 \quad(j=1, \ldots, n) .
\end{array}\right.
$$

Suppose that $m=\inf _{\mu} y$. Then from (4)

$$
\Omega(\alpha)>\exp (m)
$$

for any totally positive $\alpha$ not on the list. Since for any totally real $\alpha \neq 0, \alpha^{2}$ is of course totally positive, and $\Omega(\alpha)=\Omega\left(\alpha^{2}\right)^{1 / 2}$, so that

$$
\Omega(\alpha) \geqslant \exp \left(\frac{1}{2} m\right)
$$

for any totally real $\alpha$, with $\alpha^{2}$ not on the list.

One can in fact show that there is a minimal distribution $\mu_{\min }$ with $y=m$ (although we do not need this fact). Depending on the particular list, the minimal distribution $\mu_{\min }$ may or may not be of the form $\mu_{\alpha}$ of (2) for some totally positive $\alpha$. If it is of the form $\mu_{\alpha}$, then the method gives the least value of $\Omega(\alpha)$ for $\alpha$ not on the list, and so serves to find new small elements of $\mathcal{L}$. This is the case for instance when the list consists of $\{0,1\}$. In general, however, $\mu_{\min }$ need not be of the form $\mu_{\alpha}$. Computation indicates that this is the case when we take the list to be

$$
\left\{0,1, \beta_{1}^{2}, \beta_{2}^{2}, \beta_{3}^{2}, \alpha_{7}^{2}, \alpha_{7}^{-2}, \alpha_{60}^{2}, \alpha_{60}^{-2}\right\},
$$

which we shall use for the proof of the theorem. (Here $\alpha_{60}=2 \cos (2 \pi / 60)$, and it seems likely that $\Omega\left(\alpha_{60}\right) \approx 1.3113$ is the fifth smallest element of $\mathcal{L}$.) We then show that

$$
\Omega(\alpha) \geqslant 1.31040
$$

for totally real $\alpha$ with $\alpha^{2}$ not on (8).

3. Dualizing the Problem. It is possible to solve (5) to considerable accuracy by approximating it by a linear programming problem with a finite number of variables. However, it is much simpler to consider the dual problem to (5), which is

$$
\underset{c_{1}, \ldots, c_{n}>0}{\operatorname{Maximize}} \underset{x>0}{\operatorname{Min}} g(x, \mathrm{c}),
$$

where

$$
g(x, \mathrm{c})=\log _{+} x-\sum_{j=1}^{n} c_{j} \log \left|P_{j}(x)\right| .
$$


To avoid having to quote duality results between (5) and (10), it is sufficient for our purposes to simply note that, if $M$ is the maximum for (10), attained for $c_{i}=c_{i}^{*}$ $(i=1, \ldots, n)$, then $\min _{x>0} g\left(x, \mathrm{c}^{*}\right)=M$ so that

$$
\int_{0}^{\infty} \log _{+} x d \mu(x) \geqslant M+\sum_{j=1}^{n} c_{j}^{*} \int_{0}^{\infty} \log \left|P_{j}(x)\right| d \mu(x)
$$

for any distribution $\mu$. In particular

$$
\int_{0}^{\infty} \log _{+} x d \mu(x) \geqslant M
$$

for any $\mu$ satisfying the constraints of (5). Since there clearly exist feasible solutions to (5) (just take $\mu$ having all its weight at a large value of $x$ ), it follows that, for the minimum value $m$ of (5), $m \geqslant M$, so that (6) and (7) hold with $m$ replaced by $M$.

4. Solving the Dual Problem. To solve (10) for the problem defined by the list (8) (i.e., $n=9$, and $P_{i}(i=1, \ldots, 9)$ as the minimal polynomials of the elements of (8)), we proceeded as follows

(a) Choose a finite set $X$ of positive numbers on which $g(x, c)$ is likely to be small. A natural choice for $X$ is the set of all points midway between two consecutive zeros of $\Pi_{i=1}^{n} P_{j}(x)$.

(b) Solve the standard linear programming problem

$$
\underset{c_{1}, \ldots, c_{n}>0}{\operatorname{Maximize}} \underset{x \in X}{\operatorname{Min}} g(x, \mathbf{c}),
$$

obtaining the maximum $M_{X}$ for $c_{i}=c_{i}^{X}(i=1, \ldots, n)$. Clearly $M_{X}>M$.

(c) Add all the zeros of $g^{\prime}\left(x, \mathrm{c}^{x}\right)$ to $X$ (here 'denotes $\left.d / d x\right)$.

(d) Repeat (b) and (c) until $M_{X}$ stops decreasing.

It turned out that seven iterations of steps (b), (c) were sufficient to fix $M_{X}$ (= $M^{*}$ say). With the final values of $c_{i}^{X}\left(=c_{i}^{*}\right.$ say) we then found

$$
M^{\prime}=\min _{x>0} g\left(x, \mathrm{c}^{*}\right)
$$

using the zeros of $g^{\prime}\left(x, \mathrm{c}^{*}\right)$. Then $M^{\prime} \leqslant M<M^{*}$. Our actual results were

$$
M^{\prime}=0.5406821213<M<0.5406821290=M^{*}
$$

and

$$
\begin{aligned}
c_{1}^{*}=0.2038021734, & c_{5}^{*}=0.0085701947, \\
c_{2}^{*}=0.3277902688, & c_{6}^{*}=c_{7}^{*}=0.0019800436, \\
c_{3}^{*}=0.0446024611, & c_{8}^{*}=c_{9}^{*}=0.0008192943, \\
c_{4}^{*}=0.0221010719, &
\end{aligned}
$$

so that for totally real $\alpha$, with $\alpha^{2}$ not on the list (8),

$$
\Omega(\alpha) \geqslant \exp \left(\frac{1}{2} M^{\prime}\right)=1.3104113 \text {. }
$$

Although the calculations leading to (16), (17),(18) were performed using doubleprecision arithmetic, it is not possible to guarantee the accuracy of the results with certainty. Note, however, that once we have obtained the values (17) for the $c_{i}^{*}$, we can take them as given. The only calculation for which error analysis is required is the straightforward calculation of $M^{\prime}$ in (15). In the next section we describe how we repeated this calculation using interval arithmetic and found a rigorous lower 
bound for $M^{\prime}$, which gives the rigorous result (9) replacing (18). Since $\Omega\left(\beta_{i}\right)$ $(i=1,2,3)$ and $\Omega\left(\alpha_{7}\right)$ are all less than 1.31040 , this proves the theorem.

5. Use of Interval Arithmetic. We used the Interval Arithmetic Package of J. M. Yohe described in [2]. Given an interval $I$ and a real function $f$, the package enables us to say reliably that $f(I)$ belongs to an interval $I^{\prime}$. It was most convenient to use the package with intervals having single-precision endpoints, and this proved to be accurate enough for our purposes. We need the following

LEMMA. Let $f$ be a twice differentiable function on an interval I, with $f^{\prime \prime}>0$ on I. Suppose that $\theta \in I$ and that the interval $f(\theta)-\frac{1}{2}\left(f^{\prime}(\theta)\right)^{2} / f^{\prime \prime}(I)$ belongs to $[a, b]$. Then $\min _{x \in I} f(x) \geqslant a$.

Proof. By the Mean Value Theorem, there is, for any $x \in I$, a number $\xi=\xi(x)$ in $I$ such that

$$
\begin{aligned}
f(x) & =f(\theta)+(x-\theta) f^{\prime}(\theta)+\frac{1}{2}(x-\theta)^{2} f^{\prime \prime}(\xi) \\
& =\frac{1}{2} f^{\prime \prime}(\xi)\left(x-\theta+f^{\prime}(\theta) / f^{\prime \prime}(\xi)\right)^{2}+f(\theta)-\frac{1}{2}\left(f^{\prime}(\theta)\right)^{2} / f^{\prime \prime}(\xi) \\
& \geqslant f(\theta)-\frac{1}{2}\left(f^{\prime}(\theta)\right)^{2} / f^{\prime \prime}(\xi) .
\end{aligned}
$$

From our previous calculations (in noninterval arithmetic) we had obtained approximations $\theta_{i}(i=1, \ldots, 30)$ to the 30 zeros of $g^{\prime}\left(x, \mathrm{c}^{*}\right)$. Putting $I_{i}=\left[\theta_{i}-2^{-14}\right.$, $\left.\theta_{i}+2^{-14}\right]$, we checked using interval arithmetic that $g^{\prime}$ was of opposite sign at one end of $I_{i}$ from the other, so that $I_{i}$ did indeed contain a zero of $g^{\prime}$. It followed that

$$
\min _{x>0} g\left(x, \mathrm{c}^{*}\right)=\min _{i=1, \ldots, 30} \min _{x \in I_{i}} g\left(x, \mathrm{c}^{*}\right) \text {. }
$$

In order to apply the Lemma, we checked that $g^{\prime \prime}\left(x, \mathrm{c}^{*}\right)>0$ on $I_{i}$ and found intervals $\left[a_{i}, b_{i}\right]$ containing $g\left(\theta_{i}, \mathbf{c}^{*}\right)-\frac{1}{2}\left(g^{\prime}\left(\theta_{i}, \mathbf{c}^{*}\right)\right)^{2} / g^{\prime \prime}\left(I_{i}, \mathbf{c}^{*}\right)$. Then from (19) and the Lemma,

$$
\min _{x>0} g\left(x, \mathrm{c}^{*}\right) \geqslant \min _{i=1, \ldots, 30} a_{i}=M^{\prime \prime} \text { say. }
$$

Then since $M^{\prime \prime}<M^{\prime}$, we obtained $\Omega(\alpha) \geqslant \exp \left(\frac{1}{2} M^{\prime \prime}\right)$, to replace (18). This gave (9).

6. Acknowledgements. This work was performed while the author was visiting the University of British Columbia, Vancouver. I would like to thank the University of Wisconsin for making the Interval Arithmetic Package available, and Peter Madderom of the U.B.C. Computing Center for enabling me to use it at U.B.C. I would also like to thank Mike Patterson of the U.B.C. Computing Center for consultations about optimization packages.

Mathematics Department

James Cook University of North Queensland

Townsville 4811, Australia

1. C. J. SMYrt, "On the measure of totally real algebraic integers," J. Austral. Math. Soc. Ser. A, v. 30, 1980, pp. 137-149.

2. J. M. YoHE, "Software for interval arithmetic. A reasonably portable package," ACM Trans. Math. Software, v. 5, 1979, pp. 50-63. 